\title{
THE NODE SYSTEM OF EQUATIONS
}

BY

\author{
W. C. PETERSON ${ }^{1}$ AND J. J. LaRUE ${ }^{2}$
}

\begin{abstract}
The minimum number of simultaneous equations and unknowns required to describe the behavior of an electrical network is in many cases obtained by considering the independent node voltages to be the unknown variables. However, the usual techniques for writing node equations are not applicable to networks containing ideal voltage sources not incident to the reference node.

The node system of equations for general networks with ideal current and voltage sources arbitrarily located, is derived on the basis of a particular type of cut-set or segregate matrix, and a novel method for writing the node equations of such networks by inspection is presented. The application to networks containing vacuum tubes, transistors and magnetic coupling is illustrated.
\end{abstract}

The behavior of an electrical network may be completely described in terms of its driving functions, its parameters, and a linearly independent set of voltage or current variables. If one junction point or node in the network is chosen as a reference node, then it will be shown that some set of voltages between other nodes and this reference constitutes such an independent set; the corresponding set of equations for a linear network is here referred to as the node system of equations. An alternate independent set of variables is called the mesh or loop set of currents, which appear in the mesh system of equations. The number of linearly independent equations in each of these two systems is not generally the same. The node system involves the smaller number of equations and unknowns in the case of certain networks, and other advantages for the node system of equations have also been cited $(1,2){ }^{3}$

The usual techniques for writing node equations are not directly applicable to networks containing ideal voltage sources not directly connected to the reference node. Equation sets have been written involving an added current variable for each such ideal voltage source $(3,4)$, however, these sets of equations should be called hybrid systems since they contain both voltage and current variables.

This paper presents a derivation of the node system of equations in which the unknown variables are exclusively an independent set of node voltages. A new type of matrix called a node segregate matrix is introduced. It is shown that this matrix together with a description of the elements making up the network provides sufficient information

${ }^{1}$ Associate Professor of Electrical Engineering, North Carolina State College, Raleigh, N. C.

${ }^{2}$ Research Assistant, University of Michigan, Ann Arbor, Mich.

${ }^{3}$ The boldface numbers in parentheses refer to the references appended to this paper. 
to permit formulating the node system of equations. Two methods of formulating the node system of equations are described and several examples are presented to illustrate the application of these methods to different types of networks.

Throughout the paper, currents and voltages are considered to be functions of time and the self and mutual admittances to be functions of the derivative operator $p$; however for brevity the functional notation is omitted.

\section{NETWORKS AND ELEMENTS}

A network is considered to be composed of a set of interconnected elements. The type of network diagram used here is a representation of the network in which elements appear as oriented line segments, which meet at junctions or nodes. The elements are considered to be exclusively ideal voltage and current sources, and self admittances. In many cases mutual admittances couple certain self-admittance elements. These mutual admittances are indicated by writing the appropriate symbol, such as $Y_{j k}$, between the graphical symbols for the self-admittance elements $j$ and $k$ which are coupled.

A transformer is represented by a number of self-admittance elements equal to the number of coils, and it is understood that two equal bilinear mutual admittances are present for every pair of magnetically coupled coils. A vacuum tube in its small signal or incremental mode of operation is represented as two self-admittance elements with a common node, coupled by at least one non-zero unilinear mutual admittance. Similarly, a transistor in its small signal mode of operation is represented as two self-admittance elements with a common node, coupled by two unequal unilinear mutual admittances. In effect, the transformer, tube and transistor are treated as "black boxes" with terminal relations described completely by self and mutual admittances. The use of internal generators in tube or transistor representation is avoided since these are superfluous when the admittances are known. The details of the methods for expressing admittance relations for transformers and vacuum tubes are available in the literature (5). An indefinite or redundant admittance matrix (6) is useful for specifying the admittance relations for different types of transistor arrangements, and this mathematical device can also be used with electronic tube circuits.

The general types of voltage and current sources usually considered in the literature are treated here in terms of the more basic elements which are the ideal dissipationless sources in series or parallel combination with passive elements. The method is found to permit a direct analysis of the given network with no added complication or increase in number of equations or unknowns and without the use of preliminary "exchange of sources" (7). 
Each ideal voltage source connected between a pair of nodes is found to reduce by one the number of equations which would otherwise need to be solved simultaneously.

Precise identification of variables requires some method of polarity marking or orientation. An arrow marking on each element indicates directly the positive sense of element current, and the element voltage is oriented positively at the tail of the arrow. Any unoriented line segment in the diagram simply represents a short circuit.

\section{BASIC CONCEPTS}

The concept of the segregate (8), which is similar to that of the cutset (9), is fundamental to the present study. The segregate current equations for a network may be considered a generalization of the Kirchhoff current law relations for the network. Node current equations are a specialized form of segregate current equations.

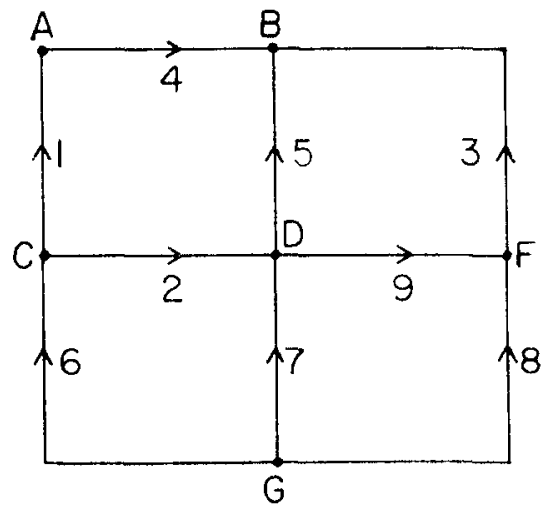

Fig. 1. A network diagram with elements represented as oriented line segments.

The network of Fig. 1 may be used to illustrate these concepts. In this diagram, the elements are numbered, but specification of element type has been intentionally omitted.

Summing the currents out of each of the nodes $B$ and $F$ yields the node current equations

and

$$
-i_{3}-i_{4}-i_{5}=0
$$

$$
i_{3}-i_{8}-i_{9}=0 \text {. }
$$

Elements four, five, eight and nine may be considered to form a segregate set (or cut-set), and the corresponding segregate current equation is

$$
-i_{4}-i_{5}-i_{8}-i_{9}=0 .
$$

It should be noted that segregate current equation (3) may be obtained by adding node current equations (1) and (2). 
Evidently other node current equations can be written for the given network. It has been shown that $(n-1)$ independent node current equations can be written for any network (10), thus since $(e)>(n-1)$, the coefficients of the linearly independent node current equations may be arranged to form an $(n-1) \times(e)$ node current matrix of rank $(n-1)$. Since segregate current equations may be formed by elementary operations on the set of node current equations, the coefficients of the segregate current equations may be arranged in the form of an $(n-1) \times(e)$ segregate current matrix which also has the rank of $(n-1)(11)$. Thus for Fig. 1, the segregate current equations result in a five row by nine column segregate current matrix of rank five.

The concept of the network tree provides a systematic method for writing a useful set of segregate current equations. In general, any set of elements which involves all of the nodes but which does not contain a closed path, constitutes a tree. For Fig. 1 for example, elements one, two, three, seven and nine form a tree.

Segregate sets may be formed by considering each element of the tree to be cut in turn thus separating the nodes of the network into two sets. The elements connecting these two sets of nodes in the original network constitute one segregate set. For example, for Fig. 1 and the chosen tree, a cut through element three separates node $B$ from the remaining nodes. In the complete network, elements three, four and five connect the two sets of nodes, leading to Eq. 1 which is a node current equation, or specialized form of segregate current equation in which one of the node sets is a single node. It is often useful to base the segregate orientation on the orientation of the cut element in the tree. Such orientation leads to a reversal of signs in Eq. 1. In the present example, a second segregate set leading to Eq. 3 results from a cut through element nine of the tree. Signs are again reversed if the orientation of element nine determines segregate orientation.

The node voltages of a network are defined as the voltages between each of $(n-1)$ nodes and the remaining or reference node, oriented toward the reference. There may however be less than $(n-1)$ independent node voltages. In particular only one node voltage associated with any subtree of ideal voltage sources in the network can be independent. As a preliminary to the development of the node equations, it is useful to augment the network by adding what will be called virtual elements located and oriented such that the voltages across these virtual elements correspond to the independent node voltages. These virtual elements are treated as zero admittance passive elements and designated by the symbol $O$ with identifying subscripts. These are not counted in the number $(e)$ of original elements.

\section{DERIVATION OF THE NODE SYSTEM}

The Kirchhoff current law and voltage law relations for a network may be respectively expressed in the form of matrix equations as 


$$
A I_{e}=0
$$

and

$$
B V_{c}=0 \text {. }
$$

These equations may be called the node current matrix equation and loop voltage matrix equation, respectively. $A$ is a node current matrix of $(n-1)$ rows, $(e)$ columns, and rank $(n-1)$. $B$ is a loop voltage matrix of $(e-n+1)$ rows, $(e)$ columns, and rank $(e-n+1)$. $I_{e}$ is a column matrix of the element currents, $(e)$ in number, and $V_{\text {. }}$. is a column matrix of element voltages, also $(e)$ in number.

It is useful to define a tree of the network, and to specify that all ideal voltage source elements are included in the tree. There may then be a number of $I_{E}$ element subtrees within the complete tree and within the network. The network is augmented by adding one type $O$ or virtual element between one arbitrarily selected node in each such subtree and the reference node, and between each of the remaining $(n-1)$ nodes and the reference. The number of virtual elements added is evidently $n_{o}=n-1-n_{e}$.

The equation corresponding to (4) for the augmented network is

$$
A_{m} I_{c m}=0
$$

where the added subscript $m$ identifies a modified matrix in each case. The modified node matrix $A_{m}$ has $(n-1)$ rows, $\left(e+n_{0}\right)$ columns and a rank the same as for the $A$ matrix, or $(n-1)$.

The union of $I_{E}$ elements and $O$ elements forms the complete network tree, and the remaining elements are called the chords. $A_{m}$ is partitioned into tree and chord submatrices, requiring a corresponding choice of element order in $I_{e m}$, and column order in $A_{m}$. Therefore Eq. 6 may be written as

where

$$
\left\|A_{T}{ }_{1}^{\prime} A_{c}\right\| I_{e m}=0
$$

$$
I_{\text {om }}=\left\|\begin{array}{ll:lll}
I_{E}^{\prime} & I_{O}^{\prime} & I_{S^{\prime}} & I_{M M^{\prime}} & H^{\prime}
\end{array}\right\|^{\prime}
$$

using the double transpose to save space.

The submatrix $A_{T}$ has been shown to have an inverse (10), therefore a new matrix may be formed by multiplying $A_{m}$ by this inverse. This matrix, called the node segregate matrix becomes

$$
\begin{aligned}
P & =A_{r}^{-1}\left\|A_{T} A_{C}\right\| \\
& =\left\|U_{T}^{\dagger} A_{T}{ }^{-1} A_{C}\right\| .
\end{aligned}
$$

The element order indicated by the first two current matrix entries in the right hand side of (8) suggests partitioning (9) so that

$$
P=\left\|\begin{array}{cc:ccc}
U_{E} & O & P_{1 S} & P_{1 M} & P_{1 I I} \\
O & U_{O} & P_{2 S} & P_{2 M} & P_{2 H}
\end{array}\right\|
$$


In the subscript notation of (8) and (10), $E$ refers to ideal voltage sources, $O$ to virtual elements, $S$ to self-admittance elements, $M$ to elements with both mutual and self admittance, and $H$ to ideal current sources. The numbers 1 and 2 refer to submatrix rows in $P$.

The product of (8) and (10) is the segregate current equation

$$
\left\|\begin{array}{cc:lll}
U_{E} & \mathrm{O} & P_{1 S} & P_{1 M} & P_{1 H} \\
O & U_{O} & P_{2 S} & P_{2 M} & P_{2 H}
\end{array}\right\|\left\|\begin{array}{c}
I_{E} \\
I_{O} \\
-- \\
I_{S} \\
I_{M} \\
H
\end{array}\right\|=0 .
$$

Since the current matrix $I_{o}$ is a null matrix, the following matrix equation is obtained from (11).

$$
P_{2 S} I_{S}+P_{2 M} I_{M}+P_{2 H} H=0
$$

or

$$
\left\|P_{2 S} \quad P_{2 M}\right\|\left\|\begin{array}{l}
I_{S} \\
I_{M}
\end{array}\right\|+P_{2 H} H=0 .
$$

An admittance equation for the self and mutual elements may be written as

$$
\left\|\begin{array}{l}
I_{S} \\
I_{M}
\end{array}\right\|=\left\|\begin{array}{cc}
Y_{S} & O \\
O & Y_{M}
\end{array}\right\|\left\|\begin{array}{l}
V_{S} \\
V_{M}
\end{array}\right\|
$$

where $Y_{S}$ is a diagonal matrix of self admittances and $Y_{M}$ is a nondiagonal matrix of self and mutual admittances for the mutual elements.

A relation for the voltages across admittance elements in terms of node voltages is now needed. To obtain this relation, consider the equation

$$
V_{e m}=P^{\prime} V_{a}
$$

where $V_{a}$ is a column matrix of $(n-1)$ entries representing an undefined set of voltage variables. Or in more detail,

$$
\left\|\begin{array}{l}
E \\
V_{O} \\
-V_{S} \\
V_{M} \\
V_{H}
\end{array}\right\|=\left\|\begin{array}{ll}
U_{E} & O \\
O & U_{O} \\
\hdashline P_{1 S^{\prime}} & P_{2 S^{\prime}} \\
P_{1 M M^{\prime}} & P_{2 M^{\prime}}^{\prime} \\
P_{1 H}^{\prime} & P_{2 H^{\prime}}^{\prime}
\end{array}\right\| V_{a}
$$

One of the relations obtainable from (15) is

$$
\left\|\begin{array}{l}
E \\
V_{o}
\end{array}\right\|=\left\|\begin{array}{ll}
U_{E} & O \\
O & U_{O}
\end{array}\right\| V_{a}
$$


which indicates that

$$
V_{a}=\left\|\begin{array}{l}
E \\
V_{0}
\end{array}\right\|=\left\|\begin{array}{ll}
i & \| \\
V_{n}
\end{array}\right\|
$$

In the last expression, $V_{o}$ has been replaced by the symbol $V_{n}$ denoting the matrix of unknown node voltages. It should be noted that there are $\left(n-1-n_{e}\right)$ entries in $V_{n}$.

Ising (16) in (15) yields the desired relation

$$
\left\|\begin{array}{l}
V_{S} \\
V_{M}
\end{array}\right\|=\left\|\begin{array}{ll}
P_{1 s^{\prime}} & P_{2 s^{\prime}} \\
P_{1 M M^{\prime}} & P_{2 M^{\prime}}
\end{array}\right\|\left\|\begin{array}{l}
E \\
V_{n}
\end{array}\right\|
$$

When matrix equations (12), (13) and (17) are combined, the result is

$$
\left\|\begin{array}{ll}
\| P_{2 S} & P_{2 M}
\end{array}\right\|\left\|\begin{array}{ll}
Y_{S} & O \\
O & Y_{M}
\end{array}\right\|\left\|\begin{array}{ll}
P_{1 S^{\prime}} & P_{2 S^{\prime}} \\
P_{1 M} M^{\prime} & P_{2 M}{ }^{\prime}
\end{array}\right\|\left\|\begin{array}{l}
E \\
V_{n}
\end{array}\right\|+P_{2 H} H=0 .
$$

Finally, performing the matrix operations indicated in (18) yields

$$
\begin{aligned}
\left\|P_{2 S} Y_{S} P_{1 S^{\prime}}+P_{2 M} Y_{M} P_{2 M}{ }^{\prime}\right\| E \\
+\left\|P_{2 S} Y_{S} P_{2 S}{ }^{\prime}+P_{2 M} Y_{M} P_{2 M}{ }^{\prime}\right\| V_{n}+P_{2 H} H=0 .
\end{aligned}
$$

The only unknown in Eq. 19 is the node voltage matrix $V_{n}$ containing $\left(n-1-n_{e}\right)$ unknown node voltage entries. The system of equations can be solved for the unknown node voltages provided that the inverse of the matrix multiplying $V_{n}$ in Eq. 19 exists. Thus the entries in $V_{n}$ represent a suitable set of independent node voltages. A non-trivial steady state solution of course requires that at least one of the entries in $E$ or $H$ be non-zero.

From the method of derivation, the node voltages are always such that the segregate current equations are satisfied; therefore Kirchhoff's current law is satisfied. However it is necessary to show that Kirchhoff's voltage law is also satisfied. That it is may be shown as follows.

An equation similar to (5) written for the augmented network is

or using Eq. 14,

$$
B_{m} V_{c m}=0
$$

$$
B_{m} P^{\prime} V_{a}=0 \text {. }
$$

But the node segregate matrix $P$ is

$$
P=A_{T^{-1}} A_{m}
$$

so that Eq. 20 becomes

$$
B_{m} A_{m}{ }^{\prime}\left\|A_{T^{-1}}\right\|^{\prime} V_{a}=0 .
$$


It can be shown that the product $B_{m} A_{m}{ }^{\prime}$ is a null matrix (12). Thus Eq. 20 is identically satisfied. But Eq. 20 contains loop voltage equation (5) for the original network, thus the loop voltage equation and Kirchhoff's voltage law are both satisfied.

The satisfaction of both of the laws of Kirchhoff assures the validity of Eq. 19. Evidently this matrix equation represents the desired node system of equations.

FORMULATING THE NODE SEGREGATE MATRIX BY INSPECTION

It was previously indicated that segregate sets of elements could be formed on the basis of cuts through the elements of a network tree. This provides a convenient method for writing the node segregate matrix $P$ so that it contains the unit submatrices indicated in Eq. 10. The method of forming the tree of ideal voltage source elements and virtual elements has been explained.

The procedure for formulating the $P$ matrix is as follows.

1. Identify the $\left(e+n_{o}\right)$ columns in matrix $P$ according to the element order in the current matrix of Eq. 11.

2. Identify the rows in matrix $P$ according to the $I_{E}$ and $O$ elements using the same order as in the first $(n-1)$ columns.

3 . Let a cut through each of the tree elements in turn define a segregate set of elements and let the orientation of the cut element determine the orientation of the segregate set. There are $(n-1)$ such segregate sets.

4. Let each segregate set determine the entries in one row of matrix $P$. Enter a $(+1)$ in a particular column if the element identifying that column is an element of the segregate, with relative orientation the same as that of the defining element, $(-1)$ for the same case except opposite orientation, and zero if the column element is not an element of the segregate.

The submatrices in matrix $P$ are evidently defined according to the element grouping in the rows and columns as indicated above and in Eq. 10. When one type of element is not present in a network, the corresponding submatrix entries in $P$ and $I_{e}$ may be omitted.

\section{EXAMPLES USING MATRIX OPERATIONS}

As a first example to demonstrate the methods thus far set forth, the node system of equations will be developed for a network based on Fig. 1. Let elements one, two and three be $I_{E}$ elements; four, five, six, seven and eight be self-admittance elements; and let element nine be an $I_{H}$ element. Node $G$ is chosen as the reference node, and $D$ and $F$ as independent nodes. 
In view of the choice of independent nodes, the tree of $I_{E}$ and $O$ elements will take the form indicated in Fig. 2.

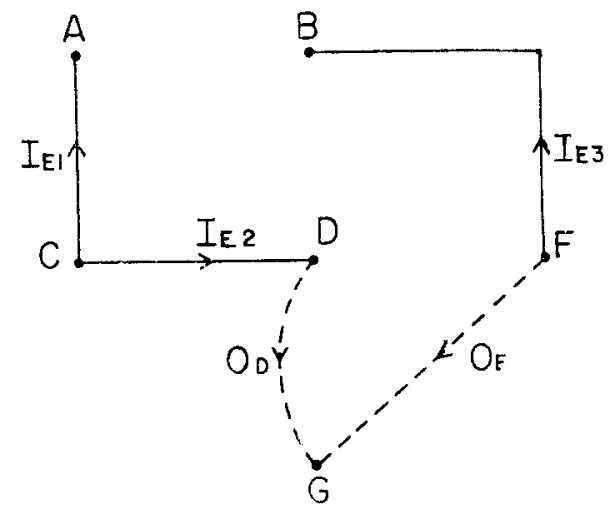

IIIG. 2. A tree for the network of Iig. 1 with specified types of elements.

The segregate sets defined by this tree determine the following form for the $P$ matrix. The submatrices $P_{1 M}$ and $P_{2 M}$ are omitted since the network involves no mutual admittances.

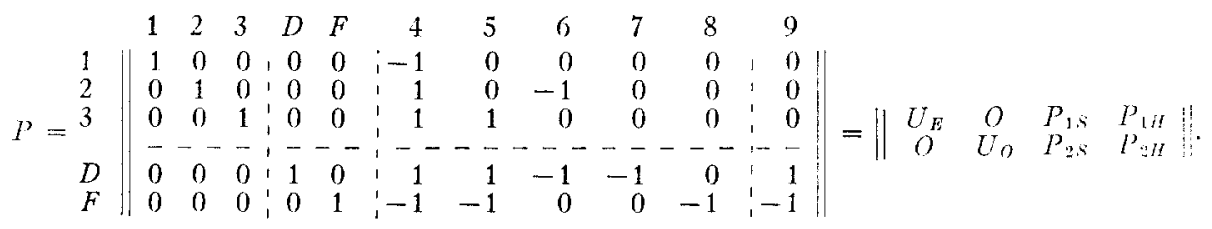

The element defining each segregate is listed at the left-hand end of the appropriate segregate row of $P$.

The node system of equations may now be obtained by performing the matrix operations indicated in Eq. 19. The submatrices $P_{1 S}, P_{2 \text { s }}$ and $P_{2 H}$ in the node segregate matrix may be easily identified, and in this example, submatrices $P_{1 M}$ and $P_{2 M}$ are null matrices. The row order in $P$, and the order of columns corresponding to self-admittance elements indicate that the voltage and self-admittance matrices are

$$
E=\left\|\begin{array}{l}
e_{1} \\
e_{2} \\
e_{3}
\end{array}\right\|, \quad V_{n}=\left\|\begin{array}{l}
y_{D} \\
v_{F}
\end{array}\right\|, \quad \text { and } \quad Y_{S}=\left\|\begin{array}{ccccc}
Y_{4} & 0 & 0 & 0 & 0 \\
0 & Y_{5} & 0 & 0 & 0 \\
0 & 0 & Y_{6} & 0 & 0 \\
0 & 0 & 0 & Y_{7} & 0 \\
0 & 0 & 0 & 0 & Y_{8}
\end{array}\right\| .
$$

The partitioning in $P$ indicates that the current matrix $H$ is a single row matrix, with the single entry $h_{9}$.

The indicated matrix operations yield the following node system of equations.

$$
\left\|\begin{array}{ccc}
-Y_{4} & Y_{4}+Y_{6} & Y_{4}+Y_{5} \\
Y_{4} & -Y_{4} & -\left(Y_{4}+Y_{5}\right)
\end{array}\right\|\|\| \begin{gathered}
e_{1} \\
e_{2} \\
e_{3}
\end{gathered}\|+\| \begin{array}{cc}
Y_{4}+Y_{5} & -\left(Y_{5}+Y_{5}\right) \\
+Y_{6}+Y_{7} & \\
-\left(Y_{4}+Y_{5}\right) & Y_{4}+Y_{5}+Y_{8}
\end{array}\|\|\left|\begin{array}{c}
v_{D} \\
v_{F}
\end{array}\|+\| \begin{array}{c}
h_{9} \\
-h_{9}
\end{array}\right|=0
$$


This node system of equations may be solved by any standard method. The independent node voltages $v_{D}$ and $v_{F}$ are thus determined in terms of the network parameters and the specified values associated with the ideal sources. The remaining node voltages can be easily determined.

As a second example consider the network of Fig. 3, containing mutual magnetic coupling between elements five and six. The mutual admittances between five and six are $Y_{56}=Y_{65}$, and the self admittances are $Y_{55}$ and $Y_{66}$. In view of the six nodes and four ideal voltage sources, only one node voltage is independent.

The admittance equation for the mutual elements is

$$
I_{M}=Y_{M} V_{M}
$$

or

$$
\left\|\begin{array}{c}
i_{5} \\
i_{6}
\end{array}\right\|=\left\|\begin{array}{ll}
Y_{55} & Y_{56} \\
Y_{65} & Y_{66}
\end{array}\right\|\left\|\begin{array}{c}
v_{5} \\
v_{6}
\end{array}\right\| .
$$

Taking node $G$ as the reference and $v_{B}$ as the independent node voltage determines the tree made up of $I_{E}$ elements one, two, three and four and the virtual element $O_{C}$. The $P$ matrix takes the following form in which two of the submatrices are omitted in view of the absence of $I_{I I}$ elements.

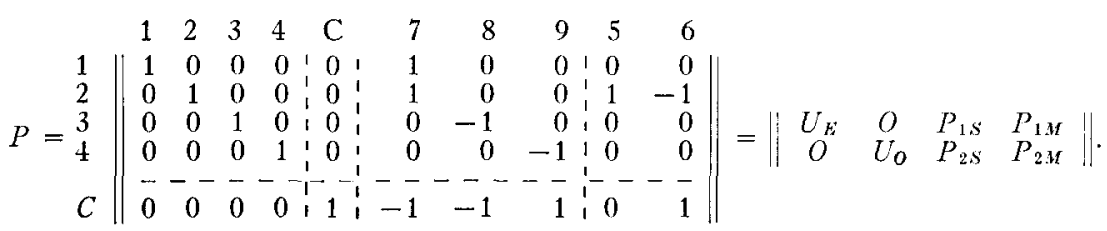

The matrix operations of Eq. 19 now yield the single node equation

$$
\begin{aligned}
-Y_{7} e_{1}+Y_{8} e_{3}-Y_{9} e_{4}-\left(Y_{7}\right. & \left.+Y_{66}-Y_{65}\right) e_{2} \\
& +\left(Y_{7}+Y_{8}+Y_{9}+Y_{66}\right) v_{C}=0 .
\end{aligned}
$$

This equation can easily be solved for the node voltage $v_{C}$ after which this value together with the specified voltage values may be used to determine the remaining node voltages.

\section{WRITING THE NODE SYSTEM BY INSPECTION}

The node system of equations may in a sense be regarded as a restatement in different form, of information contained in the network diagram. It should therefore be possible to write the node system directly by inspection of the network diagram. Methods for doing this have in fact been commonly applied to the simpler types of networks (4).

In the case of more complex networks, particularly those in which 
ideal voltage sources are not directly connected to the reference node, it is the opinion of the authors that the node system can be more accurately and easily written by inspection of the node segregate matrix $P$. Given the network diagram, the method then involves two straightforward steps: (1) writing the $P$ matrix by inspection of the diagram, and (2) writing the node system by inspection of the $P$ matrix. The first of these steps was described in a previous section.

A procedure for step (2) will now be given.

The node system of Eq. 19 may be written in the form

$$
Y_{n}\left\|\begin{array}{l}
E \\
V_{n}
\end{array}\right\|+P_{2 H} H=0
$$

where $Y_{n}$ denotes the node system admittance matrix. From Eq. 19, this is evidently the $\left(n-1-n_{\mathrm{e}}\right) \times(n-1)$ matrix

$$
Y_{n}=\left\|P_{2 S} Y_{S} P_{1 S^{\prime}}+P_{2 M} Y_{M} P_{1 M^{\prime}} P_{2 S} Y_{S} P_{2 S^{\prime}}+P_{2 M} Y_{M} P_{2 M}{ }^{\prime}\right\| \text {. }
$$

The entries in $Y_{n}$ can be determined in an orderly manner if the $(n-1)$ columns of $Y_{n}$ are first identified in the same manner as the rows of the node segregate matrix $P$, and the $\left(n-1-n_{\mathrm{e}}\right)$ rows of $Y_{n}$ in the same manner as the last $\left(n-1-n_{e}\right)$ rows of $P$, which correspond to the independent node voltages. The rules for writing the node system of equations are as follows.

1. Denote the $i^{\text {th }}$ row $j^{\text {th }}$ column entry in $Y_{n}$ by $S_{i j}$. Two cases should be considered.

(a) If $j=i, S_{i j}=S_{i i}$ is positive and is the sum of all self admittances with non-zero entries in row $i$ of the node segregate matrix $P$.

(b) If $j \neq i, S_{i j}$ is the algebraic sum of all self admittances with non-zero entries common to rows $i$ and $j$ in matrix $P$ and the mutual admittances which couple elements in row $j$ of $P$ into elements in row $i$ of $P$. The sign of an admittance term in $S_{i j}$ is positive if the two entries in $P$ corresponding to this term have the same sign and negative if they have opposite signs.

2. The entries in the column voltage matrix $\left\|E^{\prime} V_{n}^{\prime}\right\|^{\prime}$ are identified in accordance with the rows of the node segregate matrix, and in the same order. This identification corresponds with that of the columns of $Y_{n}$, and also the first $(n-1)$ columns of the node segregate matrix $P$.

3 . The entry in row $i$ of the term $P_{2 H} H$ in Eq. 24 is the sum of the $I_{I I}$ element entries in the $i^{\text {th }}$ row of $P$, each multiplied by its specified current $h$.

THE NODE SYSTEM OF EQUATIONS FOR A NETWORK CONTAINING VACUUM TUBES

As indicated previously, the small signal behavior of a vacuum tube may be specified by a matrix admittance equation (5). The self and 
mutual admittances are dependent on the type of connection, that is whether the common cathode, common plate or common grid circuit is used. Given the admittances, these can be incorporated into the node system of equations in a straightforward manner.
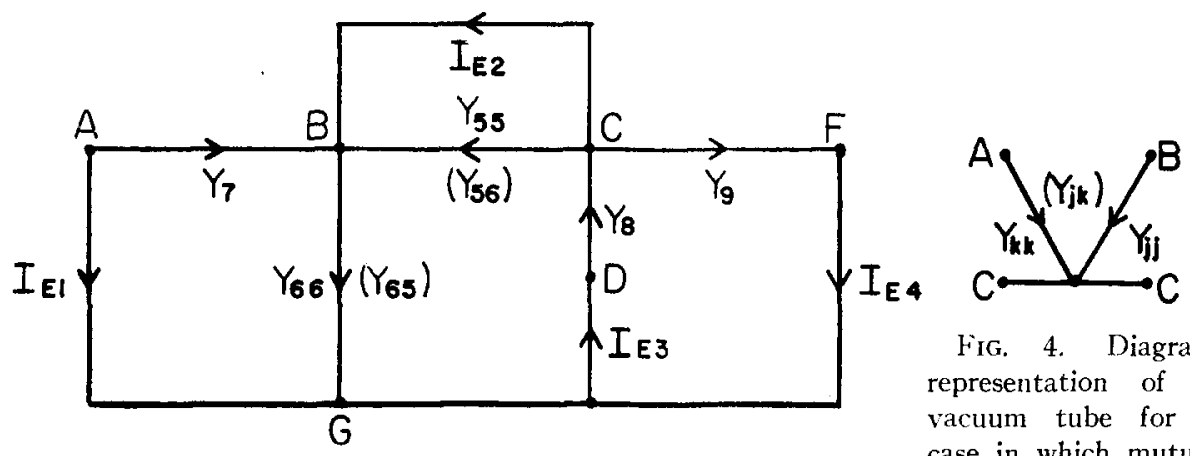

Fig. 4. Diagram representation of a vacuum tube for a case in which mutual

FIG. 3. Diagram of a network containing mutual magnetic coupling. admittance $Y_{k j}$ is zero.

The subdiagram of Fig. 4 may be used to represent the tube in the network diagram. The symbols $A$ and $B$ represent input and output nodes which are connected to the common node $C$ through the input and output self admittances $Y_{k k}$ and $Y_{j j}$, respectively. The symbol $Y_{j k}$ denotes mutual admittance between the input and output selfadmittance elements. The admittance $Y_{k j}$ is zero for low frequency operation in both the common cathode and common plate connections, but in general is not zero in the common grid case.

The matrix admittance equation corresponding to Fig. 4 in which $Y_{k j}=O$ is

$$
\left\|\begin{array}{l}
i_{k} \\
i_{j}
\end{array}\right\|=\left\|\begin{array}{ll}
Y_{k k} & O \\
Y_{j k} & Y_{j j}
\end{array}\right\|\left\|\begin{array}{c}
v_{k} \\
v_{j}
\end{array}\right\|
$$

For the common cathode case in particular, where $A$ becomes the grid, $B$ the plate and $C$ the cathode, the self and mutual admittances are

$$
\begin{aligned}
& Y_{k k}=Y_{g}, \text { the grid input self admittance, } \\
& Y_{j j}=g_{p}, \text { the plate self conductance, and } \\
& Y_{j k}=g_{M}, \text { the tube transconductance from grid to plate. }
\end{aligned}
$$

The following example shows that the node system of equations may be readily written for a network containing vacuum tubes. Such a network is shown in the diagram of Fig. 5 , in which $Y_{8}$ may be recognized as a feedback element.

Choosing node $E$ as the reference, the virtual elements needed in the tree of this network are $O_{B}, O_{C}$ and $O_{D}$, assumed to be connected be- 


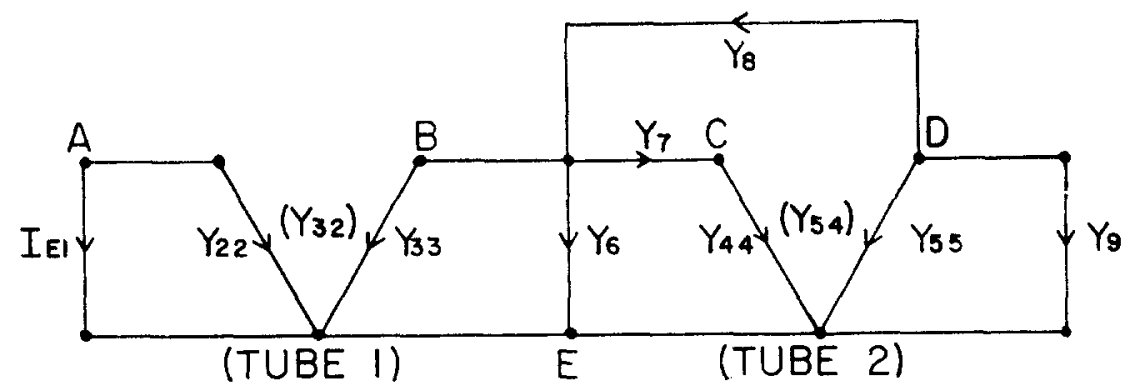

Fig. 5. Diagram of a network containing two vacuum tubes and a feedback element.

tween nodes $B, C$ and $D$, respectively, and the reference. Thesc virtual elements together with element $I_{E: 1}$ comprise the network tree.

The node segregate matrix becomes

$$
\begin{aligned}
& \begin{array}{llllllllllll}
1 & B & C & D & 6 & 7 & 8 & 9 & 2 & 3 & 4 & 5
\end{array}
\end{aligned}
$$

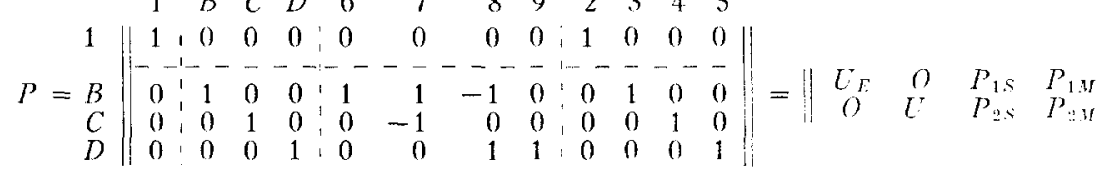

The node system of equations obtained by inspection of the node segregate matrix and knowledge that the only mutual coupling is from element two into element three and from element four into clement five is

$$
\begin{array}{ccccc}
1 & B & C & D \\
B \\
C \\
D
\end{array}\left\|\begin{array}{cccc}
Y_{6}+Y_{7} & -Y_{7} & -Y_{8} \\
Y_{32} & +Y_{8}+Y_{33} & -Y_{7} \\
0 & -Y_{7} & Y_{7}+Y_{44} & 0 \\
0 & -Y_{8} & Y_{54} & Y_{8}+Y_{9}+Y_{55}
\end{array}\right\|\|\| \begin{gathered}
e_{1} \\
v_{B} \\
v_{C} \\
v_{D}
\end{gathered} \|=0
$$

If the common cathode connection is used for each tube, the tube parameters become $Y_{33}=g_{p 1}, Y_{32}=g_{M 1}, Y_{44}=Y_{a 2}, Y_{55}=g_{p 31}$ and $Y_{54}=g_{M 2}$.

The node system of equations then takes the form

$$
\left\|\begin{array}{ccc}
Y_{6}+Y_{i}+Y_{8}+g_{p 1} & -Y_{7} & -Y_{8} \\
-Y_{i} & Y_{g 2}+Y_{7} & 0 \\
-Y_{8} & g_{M 2} & Y_{8}+Y_{9}+g_{p 2}
\end{array}\right\|\|\| \begin{gathered}
v_{B} \\
v_{C} \\
v_{D}
\end{gathered}\|=\| \begin{gathered}
-g_{M 1} e_{1} \\
0 \\
0
\end{gathered} \|
$$

It may be noted that the unilinear mutual coupling in tube two results in this example in a non-symmetrical matrix. 


\section{CONCLUSIONS}

The node system of equations has been derived from a segregate current equation for a general network. Kirchhoff's laws are both satisfied since the segregate current equation follows directly from the current law, and the node voltages satisfy the voltage law identically.

A particular form of network tree containing the ideal voltage sources and a set of virtual elements forms the basis for a direct formulation of the node segregate matrix. It was shown that the node system of equations for a complex network can readily be written by inspection of its node segregate matrix, if the element characteristics are properly considered.

It is found to be convenient to represent devices containing mutual coupling by their admittance relations as a preliminary to forming the node system of equations. This type of representation eliminates the need for voltage or current generators within the small signal equivalent circuits of vacuum tubes and transistors.

The following definition is proposed as one result of this study:

The node system of equations is a set of linearly independent simultaneous equations describing the behavior of a network, in which the unknown variables are exclusively a set of independent node voltages of the network, and in which the number of equations and independent node voltages are each equal to the number of network nodes less the number of ideal voltage sources less one.

\section{NOMENCLATURE}

$k=$ subscript denoting the $k^{\text {th }}$ network element, node or variable.

$I_{R}=$ ideal voltage source element.

$n_{e}=$ number of ideal voltage sources in a network.

$e_{k} \equiv e_{k}(t)=$ specified voltage of element $I_{E k}$.

$I_{H}=$ ideal current source element.

$n=$ number of nodes in a network.

$h_{k} \equiv h_{k}(t)=$ specifled current of element $I_{I / k}$.

$Y_{k} \equiv Y_{k}(p)=$ admittance of the $k^{\text {th }}$ element having self admittance only in mhos.

$Y_{k k} \equiv Y_{k k}(p)=$ self admittance of the $k^{\text {th }}$ element having both self and mutual admittance, in mhos.

$Y_{k j} \equiv Y_{k j}(p)=$ mutual admittance coupling the $j^{\text {th }}$ element into the $k^{\text {th }}$ element, in mhos.

$Y_{j k} \equiv Y_{i k}(p)=$ mutual admittance coupling the $k^{\text {th }}$ element into the $j^{\text {th }}$ element, in mhos.

$v_{k} \equiv v_{k}(t)=$ voltage across the $k^{\text {th }}$ element.

$i_{k} \equiv i_{k}(t)=$ current through the $k^{\text {th }}$ element, in amperes.

$e=$ number of elements in a network.

$O_{k}=$ zero admittance (virtual) element connected between node $k$ and the reference node.

$v_{A} \equiv v_{A}(t)=$ node voltage at node $A=$ voltage across virtual element $O_{A}$.

$n_{0}=$ number of type $O$ (virtual) elements in an augmented network.

$t=$ time in seconds.

$p \equiv d / d t=$ derivative operator. 


\section{REFERENCES}

(1) John W. Mrles, "Junction Analysis in Vacuum Tube Circuits," IRE Proc., Vol. 32, pp. 617-620 (1944).

(2) Hendrik W. Bode, "Network Analysis and Feedback Amplifier Design," New York, D. Van Nostrand Co. Inc., 1945, p. 16.

(3) Myril B. Reed, "Node Equations," IRE Proc., Vol. 32, pp. 355-359 (1944).

(4) Harkis A. Thompson, "A. C. Circuit Analysis," New York, McGraw-Hill Book Co., Inc., 1955, p. 115.

(5) J. S. Brown and F. D. Bennetr, "The Application of Matrices to Vacuum Tube Circuits," IRE Proc., Vol. 36, pp. 844-852 (1948).

(6) JaСOB Shekel, "Matrix Representation of Transistor Circuits," IRE Proc, Vol. 40, pp. 1493-7 (1952).

(7) M. F. Gardner and J. L. Barnes, "Transients in Linear Systems," New York, John Wiley \& Sons, Inc., 1949, p. 43.

(8) Myril B. Rise, "The Segregate, A Generalization of Kirchhoff's Current Law," NEC Proc., Vol. 13, p. 862 (1957).

(9) E. A. Guillemis, "Introductory Circuit Theory," New York, John Wiley \& Sons, Inc., 1953 , p. 17 , p. 70 .

(10) DÉnes Köntg, "Theorie der Endlichen und Unendlichen Graphen," Chelsea Publishing Co., 1950, p. 142.

(11) Garretr Birkhoff and Saunders Maclane, "A Survey of Modern Algebra," Yew York. The MacMillan Co., 1953, pp. 172-175.

(12) Myril B. Reed and Sundaram Seshu, "On Topology and Network Theory," P'rot. Symp. Circuit Analysis, Urbana, Ill., Iniv. of Illinois, 1955. 